\title{
THE NUMBER OF SQUARE-FULL DIVISORS OF AN INTEGER
}

\author{
D. SURYANARAYANA AND R. SITA RAMA CHANDRA RAO
}

ABSTRACT. Let $\alpha(n)$ denote the number of square-full divisors of $n$. In this note an asymptotic formula for $\sum_{n \leqq x} \alpha(n)$ is established.

1. Introduction. A positive integer $n>1$ is called square-full if in the canonical representation of $n$ into prime powers each prime occurs with multiplicity at least two, or equivalently, if $p$ is a prime dividing $n$, then $p^{2}$ also divides $n$. The integer 1 is also considered to be square-full. Let $L$ denote the set of square-full integers. A divisor $d>0$ of the positive integer $n$ is called square-full if $d \in L$. Let $\alpha(n)$ denote the number of square-full divisors of $n$. It is clear that $\alpha(1)=1$ and if $1<n=\prod_{i=1}^{r} p_{i}^{a_{i}}$, then $\alpha(n)=$ $\prod_{a_{i} \geqq 2}\left(a_{i}\right)$, since every square-full divisor of $n$ is a term in the expansion of the product $\prod_{a_{i} \geqq 2}\left(1+p_{i}^{2}+\cdots+p_{i}^{a_{i}}\right)$ and conversely.

Let $\beta(n)$ denote the number of divisors $d>0$ of the positive integer $n$ such that $\gamma(d)=\gamma(n)$, where $\gamma(n)$ is the core of $n$, that is, the maximal square-free divisor of $n$. It is clear that $\beta(1)=1$ and if $1<n=\prod_{i=1}^{r} p_{i}^{a_{i}}$, then $\beta(n)=\prod_{i=1}^{r}\left(a_{i}\right)$, so that $\beta(n)$ is the same as $\alpha(n)$.

The object of the present note is to establish an asymptotic formula for $\sum_{n \leqq x} \alpha(n)$. In fact, we prove

$$
\sum_{n \leqq x} \alpha(n)=\frac{\zeta(2) \zeta(3)}{\zeta(6)} x+\frac{\zeta\left(\frac{1}{2}\right) \zeta\left(\frac{3}{2}\right)}{\zeta(3)} x^{1 / 2}+\frac{\zeta\left(\frac{1}{3}\right) \zeta\left(\frac{2}{3}\right)}{\zeta(2)} x^{1 / 3}+O\left(x^{\theta}\right),
$$

where $\zeta(s)$ is the Riemann zeta function defined for $s>0, s \neq 1$, by

$$
\zeta(s)=\frac{s}{s-1}-s \int_{1}^{\infty} \frac{(t-[t])}{t^{s+1}} d t,
$$

and $\theta$ is the number which appears in the divisor problem, viz.,

$$
\sum_{n_{1} n_{2}^{2} n 3^{3} \leqq x} 1=\zeta(2) \zeta(3) x+\zeta\left(\frac{1}{2}\right) \zeta\left(\frac{3}{2}\right) x^{1 / 2}+\zeta\left(\frac{1}{3}\right) \zeta\left(\frac{2}{3}\right) x^{1 / 3}+O\left(x^{\theta}\right) .
$$

Received by the editors November 10, 1971 .

AMS 1970 subject classifications. Primary 10H25.

Key words and phrases. Square-full divisors, Riemann zeta function.

(c) American Mathematical Society 1972 
It is known that $\frac{1}{6}<\theta \leqq 20 / 69$. The left side inequality for $\theta$ is due to E. Landau (cf. [2], also cf. [3]) and the right side inequality for $\theta$ is the recent result due to E. Krätzel (cf. [1, Satz. 6]).

2. Proof of (1.1). We have $\alpha(n)=\sum_{d \delta=n ; d \in L}$ 1. Since $d \in L, d$ can be uniquely represented in the form $d=a^{2} b^{3}$, where $b$ is square-free. Hence $\alpha(n)=\sum_{d \delta=n} \sum_{a^{2} b^{3}=d} \mu^{2}(b)$, where $\mu(\mathrm{b})$ is the Möbius function. Since $\mu^{2}(b)=\sum_{e^{2} f=b} \mu(e)$, we have

Hence

$$
\alpha(n)=\sum_{d \delta=n} \sum_{a^{2} e^{6} f^{3}=d} \mu(e)=\sum_{a^{2} e^{6} f^{3} \delta=n} \mu(e) .
$$

$$
\begin{aligned}
\sum_{n \leqq x} \alpha(n)= & \sum_{a^{2} e^{\theta^{3}} \delta \leqq x} \mu(e)=\sum_{e \leqq x^{1 / 6}} \mu(e) \sum_{\delta a^{2} f^{3} \leqq x / e^{6}} 1 \\
= & \sum_{e \leqq x^{1 / 6}} \mu(e)\left\{\zeta(2) \zeta(3) \frac{x}{e^{6}}+\zeta\left(\frac{1}{2}\right) \zeta\left(\frac{3}{2}\right) \frac{x^{1 / 2}}{e^{3}}+\zeta\left(\frac{1}{3}\right) \zeta\left(\frac{2}{3}\right) \frac{x^{1 / 3}}{e^{2}}+O\left(\frac{x^{\theta}}{e^{6 \theta}}\right)\right\} \\
= & \zeta(2) \zeta(3) x \sum_{e \leqq x^{1 / 6}} \frac{\mu(e)}{e^{6}}+\zeta\left(\frac{1}{2}\right) \zeta\left(\frac{3}{2}\right) x^{1 / 2} \sum_{e \leqq x^{1 / 6}} \frac{\mu(e)}{e^{3}} \\
& +\zeta\left(\frac{1}{3}\right) \zeta\left(\frac{2}{3}\right) x^{1 / 3} \sum_{e \leqq x^{1 / 6}} \frac{\mu(e)}{e^{2}}+O\left(x^{\theta} \sum_{e \leqq x^{1 / 6}} \frac{1}{e^{6 \theta}}\right) \\
= & \zeta(2) \zeta(3) x\left\{\frac{1}{\zeta(6)}+O\left(x^{-5 / 6}\right)\right\} \\
& +\zeta\left(\frac{1}{2}\right) \zeta\left(\frac{3}{2}\right) x^{1 / 2}\left\{1 / \zeta(3)+O\left(x^{-1 / 3}\right)\right\} \\
& +\zeta\left(\frac{1}{3}\right) \zeta\left(\frac{2}{3}\right) x^{1 / 3}\left\{1 / \zeta(2)+O\left(x^{-1 / 6}\right)\right\}+O\left(x^{\theta}\right),
\end{aligned}
$$

since $6 \theta>1$.

Hence

$$
\sum_{n \leqq x} \alpha(n)=\frac{\zeta(2) \zeta(3)}{\zeta(6)} x+\frac{\zeta\left(\frac{1}{2}\right) \zeta\left(\frac{3}{2}\right)}{\zeta(3)} x^{1 / 2}+\frac{\zeta\left(\frac{1}{3}\right) \zeta\left(\frac{2}{3}\right)}{\zeta(2)} x^{1 / 3}+O\left(x^{\theta}\right),
$$

so that (1.1) follows.

\section{REFERENCES}

1. E. Krätzel, Teilerprobleme in drei Dimensionen, Math. Nachr. 42 (1969), 275-288. MR 41 \#6794.

2. E. Landau, Über die Anzahl der Gitterpunkte in gewissen Berichen (Vierte Abhandlung) Nachr. Ges. Wiss. Z. Gottingen Math.-Phys. K1. 1924, 137-150.

3. - Ausgerwählte Abhandlungen zur Gitterpunktlehre, Herausgegeben von Arnold Walfisz, VEB Deutscher Verlag der Wissenschaften, Berlin, 1962. MR 27 \#112.

Department of Mathematics, ANDhra University, Waltair, India 